\title{
Spin-Orbitronics a Novel Trend in Spin Oriented Electronics
}

\author{
Andrey A. Stashkevich ${ }^{\bowtie}$ \\ Institut Galilee, Université Sorbonne Paris Cité, Villetaneuse, France \\ 凶stachkevitch@univ-paris13.fr
}

\begin{abstract}
Introduction. The advent of spin oriented electronics, or spintronics, in the late 1980ies has not only revolutionised the very idea of contemporary electronics but has also brought about a major technological breakthrough in the field of information storage and processing. Further progress is associated with the rapidly emerging field of spinorbitronics seeking to put to maximum use the SOC (Spin-Orbit Coupling) related phenomena.
\end{abstract}

Aim. The purpose of this review paper is to outline the major trends in the dynamically developing field of spinorbitronics in the context of evolution of the mainstream spintronics. SOC related effects open up the possibility of creation of a new generation of energy saving devices, a key challenge in electronics in general.

Materials and methods. A special effort has been undertaken to make the article appealing to the general reader, especially to specialists in the field of radioelectronics and data processing. To this end, in the description of the complex physics underlying magnetic interactions preference is given to simple term "naïve" interpretations.

Results. Apart from the analysis of the fundamental features peculiar to the interfaces between ultrathin films of ferromagnetic and heavy metals and related to strong SOC, we discuss specific configurations especially promising for application-oriented research. Among others, these include spin torque microwave $(1 \ldots 50 \mathrm{GHz})$ oscillators, fast domain walls in racetrack memory and especially magnetic skyrmions.

Conclusion. Publication of this paper will facilitate creative interaction between the fundamental and applied research, thus contributing to the development of novel high-performance spintronic devices.

Keywords: spintronics, spinorbitronics, skyrmion, Spin-Orbit Coupling, spin currents, magnetic memory

For citation: Stashkevich A. A. Spin-Orbitronics a Novel Trend in Spin Oriented Electronics. Journal of the Russian Universities. Radioelectronics. 2019, vol. 22, no. 6, pp. 45-54. doi: 10.32603/1993-8985-2019-22-6-45-54

Conflict of interest. Authors declare no conflict of interest.

Submitted 12.09.2019; accepted 29.10.2019; published online 30.12.2019

Introduction. The discovery of the existence of intrinsic angular momentum inferred from the SternGerlach experiment and the introduction of the theoretical concept of electron spin by S. Goudsmit and G. Uhlenbeck in the mid-1920s had revolutionized the philosophy of the contemporary physics. It has become a fundamental cornerstone in the understanding of the atomic structure in its innumerable incarnations. At the same time, for over 60 years not a single attempt has been undertaken to make use of this useful electron property in any branch of applied physics or engineering. Most surprisingly, the electronics, that has completely transformed our world in the second half of the 20ieth century, comprising the physics, engineering, technology and applications that deal with the emission, flow and control of electrons and widely used in information processing, telecommunication, and signal processing did not show any interest in this seemingly valuable additional "degree of freedom".

Spin-polarised currents. Conventional spintronics. Characteristically, the influence of the spin on the mobility of the electrons and hence on conductivity in ferromagnetic (FM) metals, predicted by Nevill Mott as early as the mid 30ies [1] passed practically unnoticed. Experimentally it was confirmed more than thirty years later [2-4]. It took

(c) Stashkevich A. A., 2019 
another two decades to appreciate its practical importance that came with the discovery of Giant Magnetic Resistance (GMR) towards the end of the 1980s first in France by the team of Professor Albert Fert [5] and a bit later in Germany by the team of Professor P. Grünberg [6]. Not only has this outstanding scientific achievement made jointly Albert Fert and Peter Grünberg Nobel laurates in 2007 "for the discovery of Giant Magnetoresistance", but opened the door for a completely new development in electronics, spintronics, that has completely reshaped techniques for scanning data on hard disks. The application to the read heads of hard discs which appeared in 1997 has led rapidly to a colossal increase of the density of information stored in discs (from $1 \mathrm{Gbit} / \mathrm{in}^{2}$ to over $1 \mathrm{Tbit} / \mathrm{in}^{2}$ today). It should be stressed that no spintronic effect is realisable without circulation of spin-polarised current, in contrast to mainstream electronics in which the spin of the electron is ignored. Thus, at the core of the effect of GMR is a pronounced dependence of electric resistance on the spin polarity of the electron flow.

In solid state physics the exchange interaction is a fundamental microscopic quantum mechanical effect that favours electrons with parallel spins if the exchange constant is positive; this is a primary cause of ferromagnetism. Exchange coupling explains the existence of spontaneously magnetised zones referred to as magnetic domains in the absence of any magnetic field. If the constant is negative, the interaction favours electrons with antiparallel spins, potentially causing antiferromagnetism.

Spin-polarised current is instrumental in another interesting spintronic phenomenon highly useful in forming GMR related nanometric ferromagnetic structures, namely the interlayer RKKY (RudermanKittel-Kasuya-Yoshida) exchange coupling between two thin layers of magnetic materials separated by a non-magnetic spacer such as $\mathrm{Fe} / \mathrm{Cr} / \mathrm{Fe}$ or $\mathrm{Fe} / \mathrm{Au} / \mathrm{Fe}$, for example [7]. GMR was actually discovered when the exchange coupling strength and its sign was found to oscillate between ferromagnetic and antiferromagnetic as a function of the thickness of the non-magnetic metallic spacer inserted between the two ferromagnetic layers. Each ferromagnetic film is naturally magnetised via classical exchange coupling (intra-layer coupling) characterised by the so called macro-spin (collective magnetic moment of all atoms), while the mutual orientation of the two macro-spins is imposed by the inter-layer RKKY. The sign of the latter can be tuned to a desired value through an appropriate choice of the spacer thickness. Thus an antiparallel configuration of macro-spins in the FM layers can be achieved with the spacer thicknesses of about $1 \mathrm{~nm}$. The antiparallel geometry is of more importance for applications and is often called artificial or synthetic antiferromagnet.

According to theoretical analysis at the microscopic level $[8,9]$, this interlayer coupling takes place due to mobile conduction electrons playing the role of spin information carriers "informing" nuclear magnetic moments on localized inner d-shell electron spins in one FM layer on the state of magnetization in the other one. In other words, a conduction electron spin-polarised by one of the FM layers must keep its polarisation state while travelling through the spacer in the form of spin polarised current. A key criterion for choosing the right spacer is the necessity of conserving spin orientation while passing across the whole bulk of the spacer, whose thickness is typically on the order of $0.5 \ldots 2 \mathrm{~nm}$ which excludes metals with intrinsic spin diffusion length under $1 \mathrm{~nm}$. On the other hand, to minimise the number of spin - flip scattering events destroying the transport of spin due to poor film quality and numerous structural defects as a result, sophisticated film growing technologies were required which have become available in the mideighties, with the development of techniques like the Molecular Beam Epitaxy.

If one replaces a metallic spacer with an insulator one spin transfer from one FM metal layer to the other can only be implemented via electron tunnelling in which case occurs another important phenomenon in spintronics referred to as Tunnelling Magnetoresistance (TMR). The FM bilayer structures with an insulator spacer are called correspondingly Magnetic Tunnel Junctions (MTJ).

As in the case of the GMR read heads the film quality is of utmost importance. Some early observations of TMR effects in $\mathrm{Fe}-\mathrm{Ge}-\mathrm{Co}$ junctions, small and at low temperature, had been already reported by M. Jullière [10] in 1975 , but they were not easily reproducible. They could be reliably reproduced only 20 years later by the groups of Moodera and of Miyasaki on MTJ with a tunnel barrier of amorphous alumina $[11,12]$. On a laboratory scale molecular beam epitaxy is considered more reliable, while on an industrial scale the film deposition can only be done by using faster 

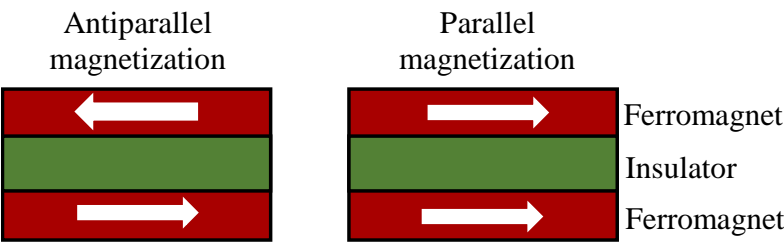

Fig. 1. Parallel and antiparallel configurations

and cheaper magnetron sputtering which is not impossible. The main idea, whatever the nature of the nonmagnetic spacer (metal or insulator), stays the same. It consists in taking advantage of a sizable difference in electric resistance between the antiparallel configuration $R_{\text {ap }}$ and the parallel one $R_{\mathrm{p}}$ corresponding to the " 1 " and "0" bits of information (Fig. 1). The size of this effect is measured by the fractional change in resistance, which is called the magnetoresistance ratio $A_{\mathrm{TMR}}=\frac{R_{\mathrm{ap}}-R_{\mathrm{p}}}{R_{\mathrm{p}}}$ Importantly, $R_{\mathrm{ap}}>R_{\mathrm{p}}$ and $A_{\mathrm{TMR}}>0$. The same applies to the GMR $A_{\mathrm{GMR}}=\frac{R_{\mathrm{ap}}-R_{\mathrm{p}}}{R_{\mathrm{p}}}$. The MTJ configuration opens up a possibility of creation of a new generation of magnetic memory called MRAM (Magnetic Random Access Memory) combining the short access time of the semiconductor-based RAM and the non-volatile character of the magnetic memories. From the point of view of these applications, MTJ has certain undeniable advantages over GMR. The main one consists in its much higher efficiency. While $A_{\mathrm{GMR}}$ typically does not exceed 15-20\%, $A_{\mathrm{TMR}}$ has reached the values as high as $500 \%$ [13]. In other words, $A_{\mathrm{TMR}} \gg A_{\mathrm{GMR}}$. The presence of an ultrathin insulator film makes the resistance of TMR elements considerably greater than in all-metal structures of GMR read heads and sensors which in certain cases simplifies the problem of impedance matching.

The ability of spin-polarized current of transferring spin angular momentum should not be overlooked either. The most important aspect of spin transfer experiments is the fact that they allow manipulating the magnetic moment of a ferromagnetic body without applying any magnetic field but only by means spin-polarised electric currents potentially leading to creation of a new generation of energy saving devices. It should be stressed that conventional control of magnetisation requires incorporation of numerous coils into the system to generate local magnetic fields to allow manipulations with specific domains which is unacceptably energy consuming.

In particular, the spin transfer via spin-polarised currents can lead to generation of magnetic torques acting on local magnetisation. This means that the state of magnetisation in memory elements can be effectively controlled electrically through the SpinTransfer Torque (STT) exerted by spin currents. Moreover, under certain conditions generation of microwave spin-wave oscillations can be realised. Magnetic dynamics in FM materials is described by the Landau-Lifshitz-Gilbert equation [14]

$$
\begin{gathered}
\frac{d \mathbf{M}}{d t}=\gamma\left(\mathbf{M} \times \mathbf{H}_{\mathrm{ef}}\right)+\frac{\alpha}{M_{0}}\left(\mathbf{M} \times \frac{d \mathbf{M}}{d t}\right)+ \\
+\frac{\beta}{M_{0}^{2}}[\mathbf{M} \times(\mathbf{M} \times \mathbf{s})],
\end{gathered}
$$

where $\mathbf{M}$ is the current value of the dynamic magnetization; $\gamma$ is the gyromagnetic ratio; $\mathbf{H}_{\mathrm{ef}}$ is the effective field including the external bias field, the dipolar magnetic field, and the Oersted field of the current; $\alpha$ is the Gilbert damping parameter; $\beta$ is the strength of the spin transfer torque proportional to the spin current density; $M_{0}$ is the saturation magnetization; and $\mathbf{s}$ is a unit vector in the direction of the spin-current polarization.

Actually, its structure describes the behaviour of a damped harmonic oscillator, which takes into account the vector features of magnetisation. Thus, the first principal term on the right-hand side (RHS) corresponds to the restoring force, rather a restoring torque, in classical mechanics determining the eigenfrequencies of eigen-oscillations in the case of lowamplitude quasi-linear magnetic dynamics. At the same time, the cross vector product appearing in this term predetermines circular polarizations of the eignmodes, thus imposing precessional temporal evolution of the dynamic magnetisation, just as in the case of ferrite based microwave devices. The second term on the RHS (the Gilbert term) describes damping (Joule heating in mechanics and electric circuits) realised as microwave losses. The effect of spin-polarized electric currents on magnetisation dynamics described by the Slonczewski's torque term (third term on the RHS) is also included into (1).

The Slonczewski's term, describing external STT pumping through spin-polarised currents, is often 


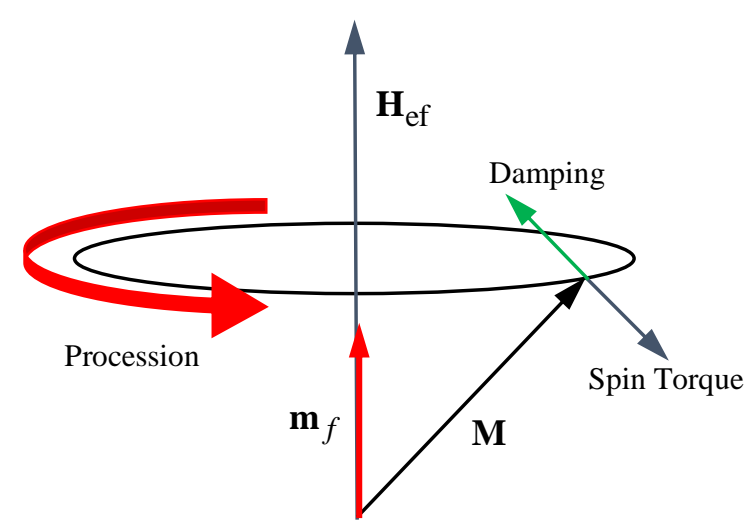

Fig. 2. Conventional Damping Torque vs Slonczewski Spin Transfer Torque

referred to as the "damping-like" torque. This means that there exist a specific orientation and a critical threshold intensity of the spin polarised current that can cancel the term describing the conventional Gilbert damping. Moreover, if this threshold level is exceeded, the sign of the effective Gilbert term is reversed and attenuation is replaced by amplification (Fig. 2) which was predicted theoretically in 1996 independently by John Slonczewski [15] and Luc Berger [16] and confirmed experimentally two years later [17]. In Fig. 2 Conventional Damping Torque (CDT) is represented by the red vector pointing towards $\mathbf{H}_{\mathrm{ef}}$ while the Slonczewski STT (Spin Transfer Torque) is given by the bleu term pointing in the opposite direction. If CDT $>$ STT, conventional damping is predominant and the angle of precession of $\mathbf{M}$ decreases, i. e. $\mathbf{M}$ tends towards $\mathbf{H}_{\mathrm{ef}}$ which is parallel to the direction of the equilibrium magnetization in the free layer defined by a unit vector $\mathbf{m}_{f}$. In the case $\mathrm{CDT}=\mathrm{STT}$, no damping occurs at all, since STT is entirely compensates CDT. Finally, the condition CDT $<$ STT corresponds to amplification of the precession which can lead to generation of microwave oscillations. This opened up a possibility of creating a unique class of nano-size oscillators in the microwave range based on generation of magnetostatic modes also known as spin-wave modes (SW) in ferromagnetic nanodot resonators. Considerable research effort has proven realizability of such devices. Typically resonance frequencies of spin transfer nano oscillators (STNO) varies from 1 to $10 \mathrm{GHz}$. Spin polarised current also exerts a "field-like" torque $\mathbf{T}_{\mathrm{FL}} \propto \beta(\mathbf{M} \times \mathbf{s})$. This torque results in a spin current-dependent frequency shift. Thus, the authors of [18] have directly verified experimentally potential double tunability of STNO demonstrating "fine" tuning of the precession frequency over a range of several gigahertz by varying the applied current and "gross" tuning with varying applied field from below 5 to above $40 \mathrm{GHz}$. At the same time, S. Bonetti et al. [19] reported a frequency as high as $46 \mathrm{GHz}$ for high magnetic fields applied normally to the film plane. The dot resonator size as a rule is around $100 \mathrm{~nm}$.

Importantly, (1) is a universal relation describing any type of magnetic dynamics, not only the case of low-amplitude spin waves. For example, the STT may be strong enough to produce magnetisation reversal to write data bits in computing [20, 21]. However, even in this case the transition from the "up" state to the "down" state takes place along a helicoidal trajectory imposed by the symmetry of the cross product in the first term on the RHS.

The concept of racetrack memory proposed in the seminal paper by the team Stuart S. P. Parkin from the IBM Almaden Research Center, San Jose [22] has become another milestone in the history of spintronics. Ultra-fast manipulating magnetic domains with spin-polarised current is an extremely appealing prospect for magnetic memory and logic with the high performance and reliability of conventional solid-state memory but at the low cost of conventional magnetic disk drive storage. The racetrack memory comprises an array of magnetic nanowires arranged horizontally or vertically on a silicon chip (Fig. 3), a step toward three-dimensional microelectronic devices [23].

The performance of such devices is completely dependent on the maximum DW velocity and so the

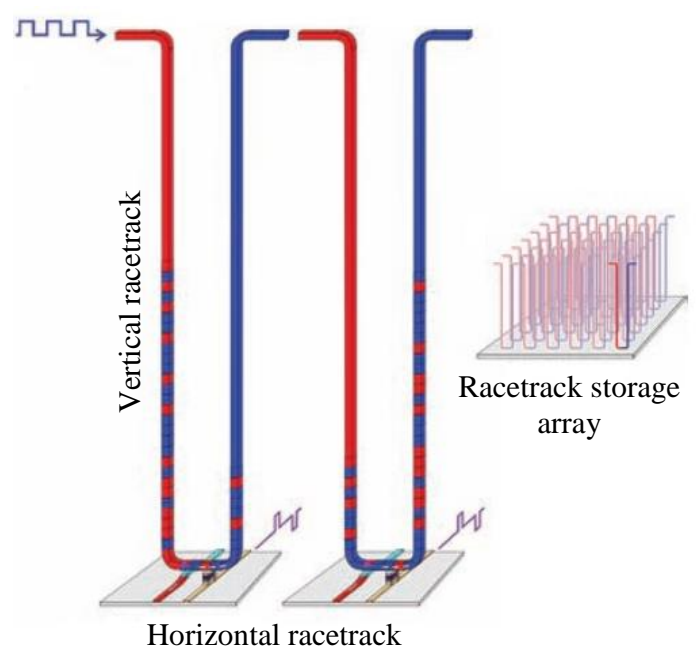

Fig. 3. Racetrack memory, a step toward three-dimensional microelectronic devices 
main question that arises is what can be done to improve this critical parameter. The answer lies in application of a wide range of effects related to SpinOrbit Coupling (SOC).

Spin-Orbit Coupling and its impact on magnetic systems. The spin-orbit interaction (SOI), also referred to as spin-orbit effect or SOC is a relativistic interaction that can be regarded as a form of effective magnetic field "seen" by the spin of the electron in its rest frame when it is moving in an external electric field typically that induced by the atomic nuclei. In other words, the electron's intrinsic spin magnetic moment is coupled to its atomic orbital magnetic moment, which leads to a number of interesting physical effects, such as shifts in electron's atomic energy levels known since the late 1920s.

The issue of SOC related phenomena had been revisited in the second half of the $20^{\text {th }}$ century, which has led to discovery of numerous original effects whose outstanding potential for information processing was realised with a considerable delay in the 1990s with the advent of spintronics. We will dwell on three of them, namely the Spin Hall Effect (SHE), Rashba Effect, and Dzyaloshinskii-Moriya Interaction (DMI), because they seem especially promising in terms of specific applications.

Since stronger electric fields are revealed in heavier atoms, it is generally considered that the SOC intensifies in the elements at the bottom of the periodic table including heavy metals, making them especially attractive for SOC related applications.

The Spin Hall effect is a transport phenomenon in which a conventional electric current in its bulk, i. e. a flow of moving charges, is transformed into a spin current, i. e. moving spins without charge flow. It was predicted by Russian physicists Mikhail I. Dyakonov and Vladimir I. Perel as early as in 1971 $[24,25]$. The term "Spin Hall Effect" was introduced much later in 1999 [26]. A clear, although somewhat naïve mechanistic analogy with a tennis ball deviating from its straight path in air in a direction depending on the direction of rotation, gives a very vivid idea of what is happening.

In a solid at the microscopic level, the deviation of conduction electrons is supposed to occur, either due to spin-dependent Mott scattering by impurities (extrinsic mechanism) or due to spin-orbit interaction as a consequence of the asymmetries in the material (intrinsic mechanism).

Both the extrinsic and the intrinsic mechanisms lead to an accumulation of spins of opposite signs on opposing lateral boundaries. In other words, a conventional charge current along the lateral direction generates a spin current in a perpendicular transverse direction leading to the above-mentioned spin accumulation. In the planar geometry, which is most practical from all points of view, this means that the conventional in-plane charge current flowing in a conducting film with strong SOC, typically a HM one, will generate an out-of-plane spin current in the vertical direction which will lead to spin accumulation on both surfaces of the HM film. The inverse SHE consists in generation of conventional charge current by a spin current in a direction perpendicular to its flow.

Unpolarised conventional charge curent $J_{\mathrm{c}}$ injected horizontally into a non magnetic material (typically a heavy metal) engenders a spin current $J_{\mathrm{S}}$ in the vertical direction. The "up" spins (red arrows) are accumulated at the NM/FM interface and are typically injected into a FM layer generting SOT within this film.

In terms of applications this HM film can be utilised as a spin current generator for more complex systems. The classical application of this approach is illustrated in Fig. 4. At the first stage, the in-plane charge current is driven in a non-magnetic HM film by an external source. Due to strong SOC it generates an out-of-plane vertical spin current that is injected into the upper FM film. The spin current thus generated and pumped into the FM film will exert a torque, referred to as Spin-Orbit Torque (SOT), on the magnetic moments in this film, similarly to the Slonczewski STT by the spinpolarised current considered previously. It should be stressed that the existence a spin current does not necessarily imply a flow of charge carrying particles which opens up the prospects for creation of devices with reduced power consumption.

Although actual thicknesses of the HM are typically small, being on the order of several nanometres (e. g.

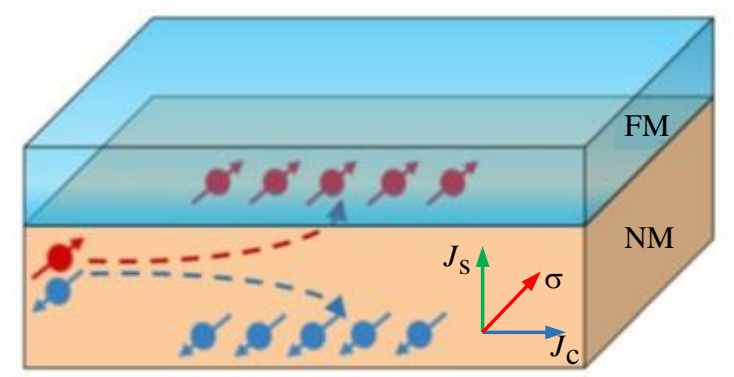

Fig. 4. Spin Hall Effect 
Известия вузов России. Радиоэлектроника. 2019. Т. 22, № 6. С. $45-54$

$\mathrm{Pt}(3 \mathrm{~nm})$ in [27], Pt (2 nm) in [28] and W (3 nm) in [29]) which corresponds to 5-10 atomic monolayers (ML), the contribution of the bulk of the film is preponderant contrary to the interfacial nature of the other two SOCrelated effects considered below.

Rashba Effect (also called Bychkov-Rashba effect). Typically, SOC effects are much stronger in low symmetry systems in reduced dimensions. Inversion symmetry is broken at interfaces, and the resultant electric field couples to the spin of itinerant electrons. This phenomenon is known as the Rashba effect.

In simple terms, somewhat naïvely, the physics of the Rashba effect boils down to the following. It can be considered as a particular case of the SOI accompanying the movement of electrons in an electric field. The conventional SOI implies fast revolution of electrons around a nucleus in its strong electric field. In the Rashba case (Fig. 5), the strong electric field is a direct result of inversion symmetry breaking at the interface, typically planar, of two different materials in the perpendicular " $z$ " direction. Due to relativistic corrections an electron moving along the " $x$ " axis with velocity $v$ in the electric field $E$ will experience in its rest frame an effective magnetic field B along " $y$ " proportional both to $v$ and $E$, thus causing precession of the electron spin in the " $y z "$ plane. This means that an external electrical voltage applied to the interface can alter the Rashba coupling strength which can be used for creation of the so-called "spin transistor" [30], although the principle of its functioning is closer to that of the electro-optic light modulator. The basic idea of this device is to rotate the spin of the injected carriers as they travel down a low-dimensional channel, connecting two ferromagnetic reservoirs (source and drain). Ideally, in the absence of the electric field, the electric spins in the vicinity of the source and of the drain are parallel, while in its presence by adjusting the gate voltage the angle of spin rotation on its trajectory from the source to the drain can be made equal to $180^{\circ}$. In other words, the initial and the final polarisations are anti-parallel. In optics this corresponds to the configuration of two parallel polarisers and an electro-optic modulator placed between them. In the "no-voltage" case the system is perfectly transparent for linearly polarised light. On the contrary, an appropriate choice of the voltage will lead to a $90^{\circ}$ rotation of the polarisation and the light will be blocked by the second polariser.

In terms of solid state physics, the presence of the Rashba SOC at the interface corresponds to the 50

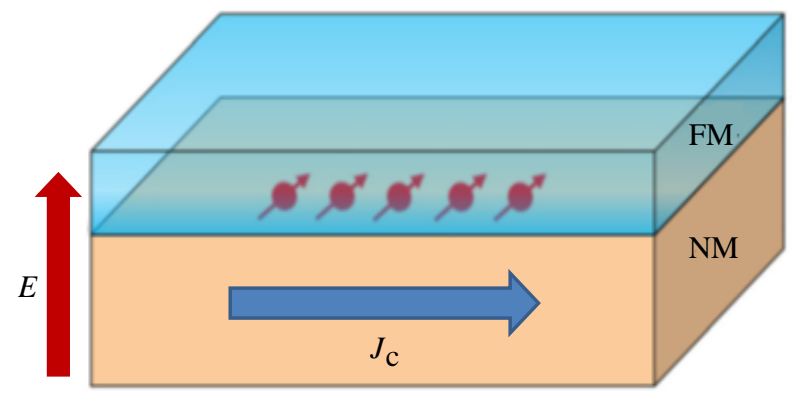

Fig. 5. Spin locking of charge current $J_{\mathrm{c}}$ via Rashba SOI

following additional term in the Hamiltonian in the form of a mixed product of the electron momentum $\mathbf{p}$, its spin polarisation $\boldsymbol{\sigma}$ and a unit vector along " $z$ "

$$
H_{R}=\alpha\left[(\boldsymbol{\sigma} \times \mathbf{p}) \mathbf{u}_{z}\right]
$$

where the coefficient $\alpha$ scales as the interface electric field $E$. The Rashba term shifts the energy balance thus favouring the in-plane orientation of electron spins as shown in Fig. 5. This phenomenon is also called the spin momentum locking. In other words a Rashba interface can be regarded as a source of spin current which leads to spin diffusion into the FM layer. Contrary to the volume nature of SHE spincurrent generation the Rashba SOC mechanisms are of purely surface configuration.

Moreover, the Rashba surface effect is at the origin of other SOC related phenomena including the interface Dzyaloshinskii-Moriya interaction (iDMI).

Interface Dzyaloshinskii-Moriya interaction (iDMI). The classical exchange interaction directly linking adjacent magnetic atoms on the microscopic quantum level is responsible for the most spectacular manifestation of the ferromagnetic ordering: the domain structure. For several decades, it was universally accepted that the exchange interactions are satisfactorily described by the isotropic Heisenberg's model favouring parallel orientation of adjacent spin which was proposed in the late 1920ies. However, thirty years later this fundamental item had been revisited and it was shown that in lowsymmetry systems lacking an inversion centre an anti-symmetric term accounting for the so-called Dzyaloshinskii-Moriya [31, 32] interaction (DMI) favouring the perpendicular configuration of neighbouring spins has to be added.

A bilayer composed of a heavy metal (HM) and a ferromagnetic metal (FM) seems to be exceptionally promising. In this structure the high SOC efficiency is ensured by the presence of a heavy metal while an interface provides the required symmetry reduction,

Spin-Orbitronics a Novel Trend in Spin Oriented Electronics 
as first suggested by A. Fert [33]. As a result, the DMI is localized in the close vicinity of the HM/FM interface and thus is known as interfacial DMI (iDMI). In this case, an "elementary iDMI cell", referred to as Fert's triangle in this paper, is seen as a triangle comprised of two magnetic atoms on the FM side linked to each other via a "SOC-carrying" atom on the HM side by means of the s-d exchange mechanism. It should be stressed that like the Rashba effect iDMI is a purely interfacial phenomenon and its role is negligible unless the thickness of the FM is very small, typically on the order of $1 \mathrm{~nm}$.

In terms of the wave science the iDMI term corresponds to an introduction of linear dispersion, producing a nonreciprocity in the propagation of spin waves in a FM film. In other words, two counterpropagating SWs with the same wavenumber will have different frequencies which can be detected by means of Brillouin Light Scattering. This technique has proved so far to be the most direct and reliable for measurements of the effective value of the intrinsic iDMI constant [34, 35]. These measurements have confirmed the plausibility of Fert's theoretical model at least for metallic FM/HM bi-layers and have identified the most efficient combinations, namely cobalt and its alloys as a ferromagnet and platinum as a heavy metal [36, 37].

However, this description is no longer relevant at interfaces between a FM metal and a dielectric material, e. g. in the $\mathrm{FeCoB} / \mathrm{TaO}_{x}$ configuration [38]. In this case the origin of iDMI is normally ascribed to the Rashba mechanism. It was shown recently theoretically that if the Rashba term (2) is added to the Hamiltonian for electronic properties of the interface region between the ferromagnetic and nonmagnetic layers chirality is introduced into the behaviour of the electrons which engenders iDMI [39]. Since the Rashba effect scales as the effective electric field at the interface, intuitively it is clear that it will be most pronounced if the "contrast" between the two constituent materials is maximal which applies to a metal-isolator combination. Moreover, in [38] it was shown that the iDMI strength can be effectively controlled by an external electric field. This too points to the Rashba related mechanisms which are very sensitive to a gate voltage. This discovery verifying the possibility of effective control of the iDMI strength electrically is very important for potential applications.

Regarding the applications, iDMIs are instrumental in stabilisation of the inner structure of the domain wall (DW) with a preferred chirality and can induce chiral structures such as skyrmions [40]. Chirality "engineering" is critically important for spinorbitronic mechanisms in high-speed DW dynamics. In ultrathin FM films, the DW spin arrangement of the Bloch type is favoured magnetostatically. However, in the Block DW configuration the damping-like torque exerted by the efficient SOT mechanisms is zero. High domain wall velocities therefore require a Néel-type domain wall. This can be obtained only in the presence of an interfacial Dzyaloshinskii-Moriya interaction (IDMI) across the interface [40], which has been verified experimentally. Thus DW velocities exceeding $0.5 \mathrm{~km} / \mathrm{s}$ have been observed in a $20 \AA \mathrm{TaN} / 15 \AA \mathrm{Pt} / 3 \AA \mathrm{Co} / 7 \AA \mathrm{Ni} / 1.5 \AA \mathrm{Co} / 50 \AA \mathrm{TaN}$ multilayer [40]. Moreover, recently a current-driven domain wall motion with a speed of $1.3 \mathrm{~km} / \mathrm{s}$ $\mathrm{Pt} / \mathrm{Gd}_{44} \mathrm{Co}_{56} / \mathrm{TaO}_{x}$ has been realised by an international team lead by Professor G. S. D. Beach from MIT [41].

Magnetic skyrmions can be described as local whirls of the spin configuration in a magnetic material (Fig. 6). Topologically they resemble magnetic cylindrical bubble domains which were intensively investigated fifty years ago with the aim of creation of non-volatile magnetic computer memory based on micrometric ferrite films using each bubble domain of micrometric size as one bit of data [42]. The fundamental difference between the bubble domains and skyrmions lies in the fact that the nanometric skyrmions can be stabilised in ultra-thin FM films $(1 \ldots 5 \mathrm{~nm})$ only in the presence of the iDMI [43].

Due to this skyrmions can be extremely small and behave as particles that can be moved, created and annihilated, which makes them promising for applications in information storage and logic technologies. Until recently, skyrmions had been

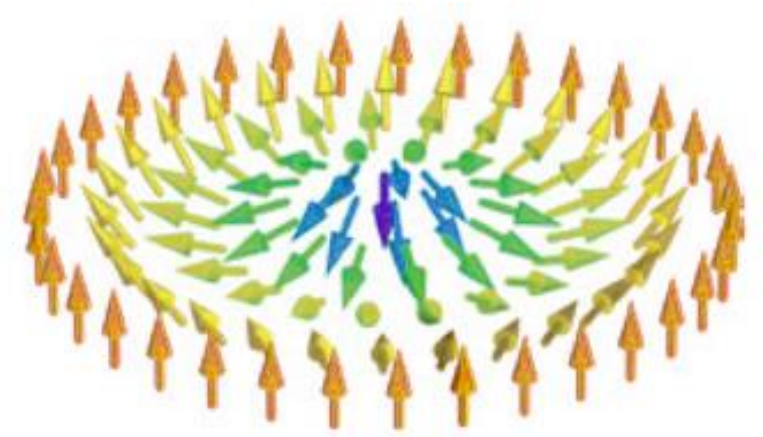

Fig. 6. Neel type skyrmion, a fast ultra-small magnetic "particle" that can be moved, created and annihilated 
observed only at low temperature and, in most cases, under large applied magnetic fields. An intense research effort has led to the identification of optimal multilayer structures in which skyrmions are stable at room temperature and can be manipulated by electrical currents. Thus in [44] skyrmions with a diameter of $130 \mathrm{~nm}$ are reported in a $\mathrm{Pt} / \mathrm{Co}(1 \mathrm{~nm}) / \mathrm{MgO}$ structure at room temperature. Smaller $50 \mathrm{~nm}$ skyrmions were observed in ultrathin (under $1 \mathrm{~nm}$ ) $\mathrm{Ir} / \mathrm{Fe} / \mathrm{Co} / \mathrm{Pt}$ multilayer stacks [45]. However, in the most recent paper a truly breakthrough result has been announced [41], namely skyrmions with a diameter approaching $10 \mathrm{~nm}$. Another important parameter crucial for applications is the velocities of their motion. Until now, their experimentally observed values did not considerably exceed $100 \mathrm{~m} / \mathrm{s}$. The highest value of $120 \mathrm{~m} / \mathrm{s}$ has been observed in $[\mathrm{Pt}(4.5 \mathrm{~nm}) / \mathrm{CoFeB}(0.7 \mathrm{~nm}) / \mathrm{MgO}(1.4 \mathrm{~nm})]_{15}$ stacks [46].

The DWs velocities experimentally observed so far are appreciably greater than those of the skyrmions. At the same time, theoretically skyrmions can be as fast as the DWs they are comprised of. Thus our estimation of the potential performance of spinorbitronic information processing devices, without losing plausibility, will be based on the realizability of $10 \mathrm{~nm}$ skyrmions reaching speeds of around $1 \mathrm{~km} / \mathrm{s}$.

This corresponds to a bit rate of $10^{11} \mathrm{bit} / \mathrm{s}$.

Conclusion. In this paper we have traced the evolution of spin oriented electronics, also known as spintronics, beginning from its belated but extremely dynamic start in late 1980ies that has brought about not only considerable progress in solid state physics but also a major technological breakthrough in the field of information storage and processing. The latter was mainly due to the discovery of the effects of Giant Magnetoresistance and Tunnel Magnetoresistance and subsequent rapid development of highly efficient magnetic read heads of hard disk drives and memory cells in magnetoresistive random access memory. In terms of solid state physics, it is the concept of spin-polarised current that plays the decisive role in the description of the above mentioned phenomena. Moreover, the spin-polarised currents are capable of transferring magnetic torques which opens a possibility of creation of spin-torque nano-oscillators (typical size $100 \mathrm{~nm}$ ) which can generate microwave signals in the frequency range from 1 to $50 \mathrm{GHz}$.

Realisation of the importance of the Spin-Orbit Coupling in spintronics and the related applications which occurred in early 2010s has become a new milestone in the development of spin oriented electronics referred to as spinorbitronics. It has triggered a new wave of breakthrough research seeking to put to maximum use the SOC related phenomena such as the Rashba effect, Spin Hall Effect and Dzyaloshinskii-Moriya interaction. On the one hand, this allows considerable amelioration of already existing devices, e. g. spin-torque nano-oscillators. On the other hand, novel purely spinorbitronic configurations have been proposed, ultra-fast domain walls for race-track memories and skyrmions being among the most promising. Skyrmions are very small $(10 \mathrm{~nm})$ and very fast $(1 \mathrm{~km} / \mathrm{s})$ cylindrical magnetic bubble domains which can be used as one bit of data. They behave as particles that can be moved, created and annihilated, which makes them promising for applications in information storage and logic technologies.

\section{REFERENCES}

1. Mott N. F. The Electrical Conductivity of Transition Metals. Proc. Roy. Soc. A. 1936, vol. 153, iss. 880, pp. 699-717. doi: 10.1098/rspa.1936.0031

2. Fert A., Campbell I. A. Two-Current Conduction in Nickel. Phys. Rev. Lett. 1968, vol. 21, iss. 16, pp. 11901192. doi: 10.1103/PhysRevLett.21.1190

3. Loegel B., Gautier F. Origine de la resistivite dans le cobalt et ses alliages dilues. J. Phys. Chem. Sol. 1971, vol. 32, iss. 12 , pp. 2723-2735. doi: 10.1016/S00223697(71)80364-5

4. Fert A., Campbell I. A. Transport Properties of Ferromagnetic Transition Metals. J. de Physique. Colloque C 1. 1971, vol. 32, no. 2-3, pp. C1-46-C1-50.

5. Baibich M. N., Broto J. M., Fert A., Nguyen Van Dau F., Petroff F., Etienne P., Creuzet G., Friederich A., Chazelas J. Giant Magnetoresistance of (001)Fe/(001) Cr Magnetic
Superlattices. Phys. Rev. Lett. 1988, vol. 61, iss. 21, pp. 2472-2475. doi: 10.1103/PhysRevLett.61.2472

6. Binash G., Grünberg P., Saurenbach F., Zinn W. Enhanced Magnetoresistance in Layered Magnetic Structures with Antiferromagnetic Interlayer Exchange. Phys. Rev. B. 1989, vol. 39, iss. 7, pp. 4828-4830. doi: 10.1103/PhysRevB.39.4828

7. Grünberg P., Schreiber R., Young Y., Brodsky M. B., Sowers H. Layered Magnetic Structures: Evidence for Antiferromagnetic Coupling of Fe Layers across $\mathrm{Cr}$ Interlayers. Phys. Rev. Lett. 1986, vol. 57, iss. 19, pp. 2442-2445. doi: 10.1103/PhysRevLett.57.2442

8. Bruno P., Chappert C. Oscillatory Coupling between Ferromagnetic Layers Separated by a Nonmagnetic Metal Spacer. Phys. Rev. Lett. 1991, vol. 67, iss. 12, pp. 1602-1605. doi: 10.1103/PhysRevLett.67.1602 
9. Bruno P., Chappert C. Ruderman-Kittel Theory of Oscillatory Interlayer Exchange Coupling. Phys. Rev. B. 1992, vol. 46, iss. 1, pp. 261-270. doi: 10.1103/PhysRevB.46.261

10. Jullière $M$. Tunneling Between Ferromagnetic Films. Phys. Lett. A. 1975 , vol. 54, iss. 3, pp. 225-226. doi: 10.1016/0375-9601(75)90174-7

11. Moodera J. S., Kinder L. R., Wong T. M., Meservey R. Large Magnetoresistance at Room Temperature in Ferromagnetic Thin Film Tunnel Junctions. Phys. Rev. Lett. 1995, vol. 74, iss. 16, pp. 3273-3276. doi: 10.1103/PhysRevLett.74.3273

12. Miyazaki T., Tezuka N. Giant Magnetic Tunneling Effect in Fe/AlzO3/Fe Junction. J. of Magnetism and Magnetic Materials. 1995, vol. 139, iss. 3, pp. L231-L234. doi: 10.1016/0304-8853(95)90001-2

13. Lee Y. M., Hayakawa J., Ikeda S., Matsukura F., Ohno H. Effect of Electrode Composition on the Tunnel Magnetoresistance of Pseudo-Spin-Valve Magnetic Tunnel Junction with a MgO Tunnel Barrier. Appl. Phys. Lett. 2007, vol. 90, iss. 21, pp. 212507-212510. doi: 10.1063/1.2742576

14. Gurevich A. G., Melkov G. A. Magnetization Oscillations and Waves. Florida, CRC Press, 1996, 464 p.

15. Slonczewski J. C. Current-Driven Excitation of Magnetic Multilayers. J. of Magnetism and Magnetic Materials. 1996, vol. 159, iss. 1-2, pp. L1-L7. doi: 10.1016/0304-8853(96)00062-5

16. Berger L. Emission of Spin Waves by a Magnetic Multilayer Traversed by a Current. Phys. Rev. B. 1996, vol. 54, iss. 13, pp. 9353-9358. doi: 10.1103/PhysRevB.54.9353

17. Tsoi M., Jansen A. G. M., Bass J., Chiang W.-C., Seck M., Tsoi V., Wyder P. Excitation of a Magnetic Multilayer by an Electric Current. Phys. Rev. Lett. 1998, vol. 80, iss. 19, pp. 4281-4284. doi: 10.1103/PhysRevLett.80.4281

18. Rippard W. H., Pufall M. R., Kaka S., Russek S. E., Silva T. J. Direct-Current Induced Dynamics in $\mathrm{CO}_{90} \mathrm{Fe}_{10} / \mathrm{Ni}_{80} \mathrm{Fe}_{20}$ Point Contacts. Phys. Rev. Lett. 2004, vol. 92, iss. 2, art. no. 027201. doi: 10.1103/PhysRevLett.92.027201

19. Bonetti S., Muduli P., Mancoff F., Åkerman J. Spin Torque Oscillator Frequency Versus Magnetic Field Angle: the Prospect of Operation Beyond $65 \mathrm{GHz}$. Appl. Phys. Lett. 2009, vol. 94, iss. 10, p. 102507. doi: 10.1063/1.3097238

20. Myers E. B., Ralph D. C., Katine J. A., Louie R. N., Buhrman R. A. Current-Induced Switching of Domains in Magnetic Multilayer Devices. Science. 1999, vol. 285, iss. 5429, pp. 867-870. doi: 10.1126/science.285.5429.867

21. Katine J. A., Albert F. J., Buhrman R. A., Myers E. B., Ralph D. C. Current-Driven Magnetization Reversal and Spin-Wave Excitations in Co/Cu/Co Pillars. Phys. Rev. Lett. 2000, vol. 84, iss. 14, pp. 3149-3152. doi: 10.1103/ PhysRevLett.84.3149

22. Parkin S. S. P., Hayashi M., Thomas L. Magnetic Domain-Wall Racetrack Memory. Science. 2008, vol. 320, iss. 5873, pp. 190-194. doi: 10.1126/science.1145799
23. Lavrijsen R., Lee J.-H., Fernandez-Pacheco A., Petit D. C. M. C., Mansell R., Cowburn R. P. Magnetic Ratchet for Three-Dimensional Spintronic Memory and Logic. Nature. 2013, vol. 493, iss. 7434, pp. 647-650. doi: 10.1038/nature11733

24. Dyakonov M. I., Perel V. I. Possibility of Orientating Electron Spins with Current. JETP Lett. 1971, vol. 13, iss. 11, pp. 467-469.

25. Dyakonov M. I., Perel V. I. Current-Induced Spin Orientation of Electrons in Semiconductors. Phys. Lett. A. 1971 , vol. 35, iss. 6, pp. 459-460. doi: 10.1016/03759601(71)90196-4

26. Hirsch J. E. Spin Hall Effect. Phys. Rev. Lett. 1999, vol. 83, iss. 9, pp. 1834-1837. doi: 10.1103/PhysRevLett.83.1834

27. Emori S., Bauer U., Sung-Min Ahn, Martinez E., Beach G. S. D. Current-Driven Dynamics of Chiral Ferromagnetic Domain Walls. Nature Materials. 2013, vol. 12, iss. 7, pp. 611-616. doi: 10.1038/nmat3675

28. Qiu Xuepeng, Narayanapillai K., Wu Yang, Deorani P., Yang Dong-Hyuk, Noh Woo-Suk, Park Jae-Hoon, Lee Kyung-Jin, Lee Hyun-Woo, Yang Hyunsoo. Spin-OrbitTorque Engineering via Oxygen Manipulation. Nature Nanotechnology. 2015, vol. 10, iss. 4, pp. 333-338. doi: 10.1038/NNANO.2015.18

29. Kim Gyu Won, Samardak A. S., Kim Yong Jin, Cha In Ho, Ognev A. V., Sadovnikov A. V., Nikitov S. A., Kim Young Keun. Role of the Heavy Metal's Crystal Phase in Oscillations of Perpendicular Magnetic Anisotropy and the Interfacial Dzyaloshinskii-Moriya Interaction in W/Co-Fe-B/MgO Films. Physical Review Applied. 2018, vol. 9, iss. 6, art. no. 064005 . doi: 10.1103/PhysRevApplied.9.064005

30. Datta S., Das B. Electronic Analog of the ElectroOptic Modulator. Appl. Phys. Lett. 1990, vol. 56, iss. 7, pp. 665-667. doi: 10.1063/1.102730

31. Dzyaloshinskii I. E. Thermodynamic Theory of "Weak" Ferromagnetism in Antiferromagnetic Substances. JETP. 1957, vol. 5, no. 6, p. 1259.

32. Moriya T. New Mechanism of Anisotropic Superexchange Interaction. Phys. Rev. Lett. 1960, vol. 4, iss. 5, pp. 228-230. doi: 10.1103/PhysRevLett.4.228

33. Fert A. Magnetic and Transport Properties of Metallic Multilayers. Materials Science Forum. 1990, vols. 5960, pp. 439-480. doi: 10.4028/www.scientific.net/MSF.5960.439

34. Stashkevich A. A., Belmeguenai M., Roussigné Y., Cherif S. M., Kostylev M., Gabor M., Lacour D., Tiusan C., Hehn M. Experimental Study of Spin-Wave Dispersion in Py/Pt Film Structures in the Presence of an Interface Dzyaloshinskii-Moriya interaction. Phys. Rev. B. 2015, vol. 91, iss. 21, art. no. 214409. doi: 10.1103/PhysRevB.91.214409

35. Nembach H. T., Shaw J. M., Weiler M., Jué E., Silva T. J. Linear Relation between Heisenberg Exchange and Interfacial Dzyaloshinskii-Moriya Interaction in Metal Films. Nature Physics. 2015, vol. 11, iss. 10, pp. 825-829. doi: $10.1038 /$ nphys3418

36. Belmeguenai M., Adam J.-P., Roussigné Y., Eimer S., Devolder T., Kim Joo-Von, Cherif S. M., Stashkevich A., 
Известия вузов России. Радиоэлектроника. 2019. Т. 22, № 6. С. 45-54

Journal of the Russian Universities. Radioelectronics. 2019, vol. 22, no. 6, pp. 45-54

Thiaville A. Interfacial Dzyaloshinskii-Moriya Interaction in Perpendicularly Magnetized $\mathrm{Pt} / \mathrm{Co} / \mathrm{AlO}_{x}$ Ultrathin Films Measured by Brillouin Light Spectroscopy. Phys. Rev. B. 2015, vol. 91, iss. 18, art. no. 180405(R). doi: 10.1103/ PhysRevB.91.180405

37. Belmeguenai M., Gabor M. S., Roussigné Y., Stashkevich A., Chérif S. M., Zighem F., Tiusan C. Brillouin Light Scattering Investigation of the Thickness Dependence of Dzyaloshinskii-Moriya Interaction in $\mathrm{CO}_{0.5} \mathrm{Fe}_{0.5}$ Ultrathin Films. Phys. Rev. B. 2016, vol. 93, iss. 17, art. no. 174407. doi: 10.1103/PhysRevB.93.174407

38. Srivastava T., Schott M., Juge R., Křižákov V., Belmeguenai M., Roussigné Y., Bernand-Mantel A., Ranno L., Pizzini S., Chérif S.-M., Stashkevich A., Auffret S., Boulle O., Gaudin G., Chshiev M., Baraduc C., Béa H. Large-Voltage Tuning of Dzyaloshinskii-Moriya Interactions: A Route toward Dynamic Control of Skyrmion Chirality. Nano Lett. 2018, vol. 18, iss. 8, pp. 4871-4877. doi: 10.1021/ acs.nanolett.8b01502

39. Kim Kyoung-Whan, Lee Hyun-Woo, Lee Kyung-Jin, Stiles M. D. Chirality from Interfacial Spin-Orbit Coupling Effects in Magnetic Bilayers. Phys. Rhys. Lett. 2013, vol. 111, iss. 21, art. no. 216601. doi: 10.1103/PhysRevLett.111.216601

40. Ryu K.-S., Thomas L., Yang S.-H., Parkin S. Chiral Spin Torque at Magnetic Domain Walls. Nat. Nanotech. 2013, vol. 8, iss. 7, pp. 527-533. doi: 10.1038/ nnano.2013.102

41. Caretta L., Mann M., Büttner F., Ueda K., Pfau B., Günther C. M., Hessing P., Churikova A., Klose C., Schneider M., Engel D., Marcus C., Bono D., Bagschik K., Eisebitt S., Beach G. S. D. Fast Current-Driven Domain
Walls and Small Skyrmions in a Compensated Ferrimagnet. Nature Nanotech. 2018, vol. 13, iss. 12, pp. 1154-1160. doi: 10.1038/s41565-018-0255-3

42. De Leeuw F. H. Physical Principles of Magnetic Bubble Domain Memory Devices. Ed. by W. E. Proebster. Digital Memory and Storage. Vieweg, 1978, pp. 203-215.

43. Fert A., Cros V., Sampaio J. Skyrmions on the Track. Nature Nanotech. 2013, vol. 8, iss. 3, pp. 152-156. doi: $10.1038 /$ nnano.2013.29

44. Boulle O., Vogel J., Yang H., Pizzini S., Chaves D. de Souza, Locatelli A., Menteş T. Onur, Sala A., BudaPrejbeanu L. D., Klein O., Belmeguenai M., Roussigné Y., Stashkevich A., Chérif S.-M., Aballe L., Foerster M., Chshiev M., Auffret S., Miron I. M., Gaudin G. Room-Temperature Chiral Magnetic Skyrmions in Ultrathin Magnetic Nanostructures. Nature Nanotech. 2016, vol. 11, iss. 5, pp. 449-454. doi: 10.1038/nnano.2015.315

45. Soumyanarayanan A., Raju M., Gonzalez Oyarce A. L., Tan Anthony K. C., Im Mi-Young, Petrović A. P., Khoo Ho K. H., Tran M., Gan C. K., Ernult F., Panagopoulos C. Tunable Room-Temperature Magnetic Skyrmions in Ir/Fe/Co/Pt Multilayers. Nature Materials. 2017, vol. 16, iss. 9, pp. 898-904. doi: 10.1038/nmat4934

46. Woo S., Litzius K., Krüger B., Im Mi-Young, Caretta L., Richter K., Mann M., Krone A., Reeve R. M., Weigand M., Agrawal P., Lemesh I., Mawass M.-A., Fischer P., Kläui M., Beach G. S. D. Observation of Room-Temperature Magnetic Skyrmions And Their Current-Driven Dynamics in Ultrathin Metallic Ferromagnets. Nature Materials. 2016, vol. 15 , iss. 5, pp. 501-506. doi: 10.1038/nmat4593

\section{Information about the author}

Andrey A. Stashkevich, Dr. Sci. (Eng.) (1994), Professor (2001) of Physics, Institut Galilee, Universite Sorbonne Paris Nord. The author of more than 100 scientific publications. Area of expertise: dinamics of ferromagnetic nanostructures; magnetooptics; magnetic metamaterrials; microwave signal processing.

Address: Laboratoire des Sciences des Procedes et des Materiaux-LSPM - CNRS UPR3407, 99 avenue J. B. Clement, Villetaneuse 93 430, France

E-mail: stachkevitch@univ-paris13.fr 